\title{
Spubsibaúde
}

\section{Atuação do (a) enfermeiro (a) na prevenção e controle das infecções hospitalares na Unidade de Terapia Intensiva Neonatal}

\author{
Performance of the nurse in the prevention and control of nosocomial infections in the Neonatal \\ Intensive Care Unit
}

\author{
Bruna Rodrigues Martins de Jesus" $\bullet$
}

Faculdade Venda Nova do Imigrante, Quixeramobim, Ceará, Brasil. *Autor para correspondência. E-mail: graduando@outlook.com

Resumo: Introdução: A ocorrência de infecção hospitalar tem sido identificada como um importante problema de saúde pública no Brasil e no mundo crescente em Unidade de Terapia Intensiva Neonatal (UTIN) desenvolvendo vários temas para discussão, buscando formas de prevenção e controle dessas infecções. Nesse contexto, surge na tentativa de melhorar o serviço e minimizar os riscos de infecção neonatal, modificando e padronizado a assistência. O objetivo deste artigo foi discutir a atuação do (a) enfermeiro (a) no processo de prevenção e controle de infecção hospitalar na UTIN, com base na identificação das prioridades clínicas dos recém-nascidos (RNs). Trata-se de um estudo descritivo, através da revisão de literatura, embasado nos artigos científicos selecionados na base dos dados do LILACS, SCIELO (Scientific Eletrônico Library), BVS, Teses e Protocolos do Ministério da Saúde .Revisão: Na UTIN, que é um setor crítico, deve haver uma monitoração diária, fornecendo dados que ajudem a Comissão a analisar estatísticas mensais, além de permitir a implementação de ações preventivas para reduzir a taxa de infecção hospitalar como um todo Discussão: As discursões obtidas se dividiram em dois temas propostos: Entendendo os meios de infecções e fatores de risco na UTIN frente aos procedimentos de enfermagem e A infecção hospitalar no contexto do cuidado do (a) enfermeiro (a), orientando os profissionais de saúde e acadêmicos de enfermagem sobre a importância de tais medidas preventistas. Considerações finais: Conclui-se que o (a) enfermeiro (a) destaca-se por ser o profissional qualificado em assumir o papel de orientador e educador da equipe de enfermagem e dos usuários dos serviços de saúde, realizando de forma qualificada a tocante vigilância das infecções hospitalares em neonatos, ao caracterizar tais eventos numa abordagem local, ressaltando a importância de efetuar medidas de controle e prevenção, a fim de reduzir os riscos existentes em uma UTIN.

Palavras-chave: unidade de terapia intensiva neonatal, enfermagem neonatal, infecção hospitalar.

\begin{abstract}
Introduction: The occurrence of nosocomial infection has been identified as an important public health problem in Brazil and in the growing world in the Neonatal Intensive Care Unit (NICU), developing various topics for discussion, seeking ways to prevent and control these infections. In this context, it appears in an attempt to improve the service and minimize the risks of neonatal infection, modifying and standardizing care. The purpose of this article was to discuss the role of the nurse in the process of prevention and control of nosocomial infection in the NICU, based on the identification of the clinical priorities of newborns (NBs). This is a descriptive study, through a literature review, based on the scientific articles selected on the basis of LILACS, SCIELO (Scientific Electronic Library), VHL, Theses and Protocols from the Ministry of Health. Review: At the NICU, which is a critical sector, there should be daily monitoring, providing data that help the Commission to analyze monthly statistics, in addition to allowing the implementation of preventive actions to reduce the rate of nosocomial infection as a whole Discussion: The speeches obtained were divided into two proposed themes : Understanding the means of infections and risk factors in the NICU in face of nursing procedures and Hospital infection in the context of nurse care, guiding health professionals and nursing students on the importance of such preventive measures . Final considerations: It is concluded that the nurse stands out for being the qualified professional in assuming the role of advisor and educator of the nursing team and users of health services, performing in a qualified way the touching surveillance hospital infections in neonates, by characterizing such events in a local approach, emphasizing the importance of carrying out control and prevention measures in order to reduce the risks that exist in an NICU.
\end{abstract}

Keywords: neonatal intensive care unit, neonatal nursing, hospital infection. 


\section{Introdução}

A infecção em nivél hospitalar tem como principais agentes etiológicos as bactérias, vírus e fungos que são os precursores em altos índices de contaminação em Unidade de Terapia Intensiva Neonatal (UTIN), geradores de uma grande problemática ética e ilegal, causando grandes prejuízos às instituições, aos pacientes e a sociedade.

Nesse contexto, os objetivos do estudo foram compreender os fatores que levam a infecção hospitalar em Unidade de Terapia Intensiva Neonatal, complementando com a relação dos profissionais de saúde frente aos variados tipos de infecção, principalmente estar atentos à infecção cruzada, onde microrganismos são transmitidos por contatos diretos entre pacientes e profissionais, mãos contaminadas, objetos inanimados e os próprios visitantes, com foco em minimizar a mortalidade neonatal devido à infecção.

A Unidade de Terapia Intensiva Neonatal (UTIN), uma unidade que concentra um grupo especifico de pacientes que demanda uma necessidade de atenção especial, além dos cuidados frequentes, monitoramento 24 horas e procedimentos muitas vezes invasivos.

Cabe ressaltar o conceito de Infecção Hospitalar segundo a Portaria No 2616/98 do Ministério da Saúde, como sendo aquela adquirida após admissão do paciente e que se manifeste durante a internação ou após a alta, quando puder ser relacionada com a internação ou a procedimentos hospitalares. São também convencionadas infecções hospitalares aquelas manifestadas antes de 72 horas da internação, quando associadas a procedimentos diagnósticos e/ou terapêuticos, realizados durante este período (Sousa et al., 2008).

O controle de infecção hospitalar ou nosocomiais na UTIN é de responsabilidade multiprofissional, mas a equipe de enfermagem tem como obrigação garantir o menor número de recém-nascido com infecção hospitalar, já que são os profissionais mais próximos dos pacientes, dando assistência até a sua alta.

O período neonatal cornpreende os primeiros 28 dias de vida da enanca, Trata-se de um período de adaptacóes anatómicas e fisiológicas para o recém-nascido (RN). A imaturidade do sistema imunológico, a ;HISencia de flora própria, a prcmaturidade e o baixo peso são fatores que predispóern $~ 1$ sepse no RN. A raca negra e o sexo masculino sao fatores de risco adicionais presentes, estudados no momento atual sem cornprovacáo definitiva para sepse neonatal, que é ainda é urna das maiores causas de óbitos para os recémnascidosl (Sousa Tomaz et al., 2011).

A infecção hospitalar é complicação comum nos pacientes hospitalizados e configura-se como importante causa de morbidade e mortalidade em unidades de terapia intensiva neonatais (UTINs). A mortalidade neonatal (óbito nos primeiros 28 dias de vida) corresponde a um terço da mortalidade infantil global (Dal-Bó et al., 2012).

Observa-se que na prevenção e o controle da infecção na UTIN, os cuidados de enfermagem devem seguir um padrão individualizado, atendendo às necessidades específicas do RN e respeitando todas as regras do Controle de Infecção Hospitalar (CCIH), diminuindo, assim, o índice de mortalidade e morbidade dos recémnascidos.

É necessário à conscientização de que quanto mais cedo forem identificados os fatores de risco, melhores as condições para promover segurança ao RN.

Além de promover uma qualificada assistência, a enfermagem deve monitorar a conservação dos materiais e instrumental, registro das ocorrências referentes ao RN, bem como ao pessoal, procedimento e estratégias educacionais.

Um aspecto importante é a criação de um ambiente propício para o tratamento do recém-nascidos, livre de estímulos nocivos, que promova um crescente desenvolvimento positivo e minimize os efeitos negativos da doença e da separação dos pais.

Diante disso, nota-se a importância do cuidado constante e do olhar clínico de todo o corpo profissional da unidade neonatal, em especial da equipe de enfermagem que são os que prestam assistência 24 horas à beira do leito tornando possível: a identificação dos primeiros sinais e sintomas da $\mathrm{IH}$, qual o tipo de infecção, se hospitalar ou não, e o tratamento imediato dos recém-nascidos (RN) infectados, pois o conhecimento precoce dessas complicações pode melhorar significativamente a evolução do paciente (Almeida Jorge et al., 2013).

A escolha do tema emergiu das aulas de graduação em enfermagem sobre os riscos de infecção nos serviços de saúde, principalmente na UTIN, após muitas pesquisas em bases de dados eletrônicos informatizados verificou-se que, mesmo sendo um tema de muita relevância entre os profissionais e nas instituições de saúde, poucos estudos são realizados sobre a prevenção de controle dessas infecções pelos (as) enfermeiros (as).

O objetivo geral deste trabalho consiste em discutir a atuação do (a) enfermeiro (a) no processo de prevenção e controle das infecções hospitalares da UTIN, com base na identificação das formas de assistência, sustentado pelos objetivos específicos: Identificar os fatores de risco para a ocorrência de infecção em UTIN, 
descrever os procedimentos de enfermagem que reduzem à infecção hospitalar em UTIN e demostrar a importância da adesão dos profissionais de enfermagem nas normas e rotinas preconizadas nas UTIN.

Trata-se de um estudo descritivo, através da revisão de literatura, embasada em artigos científicos selecionados na base de dados do SCIELO, LILLACS, BVS, Teses e Protocolos do Ministério da Saúde sobre os temas selecionados entre os anos 2007 a 2017, com o intuito de compreender a importância da atuação do (a) enfermeiro (a) na prevenção e controle de infecção hospitalar neonatal, apresentando os seguintes descritores: Unidade de Terapia Intensiva Neonatal, Enfermagem Neonatal e Infecção Hospitalar.

\section{Revisão e discussão}

Na UTIN, que é um setor crítico, deve haver uma monitoração diária, fornecendo dados que ajudem a Comissão a analisar estatísticas mensais, além de permitir a implementação de ações preventivas para reduzir a taxa de infecção hospitalar como um todo.

A Comissão de Controle de Infecção Hospitalar deve possuir um vínculo estreito com a Central de Materiais de Esterilização (CME), outro processo importante na prevenção das infecções, pela esterilização de materiais.

\section{Entendendo os meios de infecções e fatores de risco na utin frente aos procedimentos de enfermagem}

O trabalho da (o) enfermeira (o) dentro de uma UTIN é um desafio diário, pois requer habilidade, vigilância, sensibilidade e respeito, pois se trata de pacientes extremamente vulneráveis e altamente dependentes da equipe que lhe está prestando assistência.

A incidência de infecções varia amplamente na UTIN dependendo de fatores ambientais e diferenças em prática clínica, os prematuros são especialmente vulneráveis a infecções nosocomiais, por causa da severidade do seu estágio de desenvolvimento e a exposição a procedimentos invasivos.

Os recém-nascidos prematuros permanecem por Iongos períodos de internacáo em Unidade de Terapia Intensiva Nccnatal (UTIN), sendo submetidos a diversos procedimentos invasivos, tais como: cateterismo arterial e venoso, nutricáo parenteral, íntubacáo traqueal, sondagem gástrica ou gastroduodenal, derivacoes ventrículo-peritoníais, drenagem torácica e ventilacáo mecânica que os expóem ainfcccáo (Sousa Tomaz et al., 2011).

Apesar dos recentes avanços na intensiva assistência, há exposição de várias complicações infecciosas como da corrente sanguínea, por infecções respiratórias e do trato urinário, idade gestacional e baixo peso ao nascer são os mais frequentes riscos individuais identificados e susceptíveis a infecção hospitalar pela sua baixa imunidade.

Ainda, de acordo com Neves et al. (2006):

O desafio é maior nas unidades de neonatologia, nas quais os processos infecciosos são os principais responsáveis pela elevada morbidade e mortalidade desse período. A IH em pacientes com sistema imunológico imaturo e com maior exposição a dispositivos invasivos é difícil de ser prevenida e controlada. Os avanços tecnológicos aumentaram a sobrevida do recém-nascido (RN) com doenças graves e prematuridade, sendo que são os maiores responsáveis pelo sucesso da manutenção da vida.

Os procedimentos invasivos são os que mais podem provocar infecção hospitalar expondo os RN ao risco, pois há o rompimento da proteção do meio externo contra o meio interno. A pele é colonizada por distintos microrganismos, devendo ser prioritária à antissepsia antes do rompimento dessa proteção, a punção venosa é muito realizada em unidades neonatais sendo comum como meio de infecção.

Um dos procedimentos invasivos como punção venosa, foi comumente apontado nos estudos como um dos procedimentos que mais representa risco para aquisição de infecção. Todavia, esse procedimento apresenta menor risco quando comparado à inserção de Cateteres, devendo ser manuseado com o mesmo rigor asséptico que os outros dispositivos centrais, pois o tempo de permanência quando comparado com outros dispositivos pode torna-se maior quando bem cuidado pela enfermagem.

Sabe-se que as mãos dos profissionais são o principal meio de transporte dessas infecções. A pele do RN é a principal porta de entrada de microrganismos patógenos, sendo que cerca de $80 \%$ dos recém -nascidos prematuramente desenvolvem alguma injúria na pele até o primeiro mês de vida e aproximadamente $25 \%$ de todos os pré-termos e de baixo peso, terão ao menos um episódio de sepse até o terceiro dia de vida (Lorenzini et al., 2013).

A enfermagem busca por obrigação aplicar e incentivar maior rigor na adoção dos princípios de antissepsia com os demais profissionais da equipe, mas durante o decorrer dos procedimentos há desatenção e 
desobediência a estes princípios pela equipe de enfermagem, sendo meio comum a contaminação de campos e cateteres.

Outro procedimento indicado como de risco foi a intubação endotraqueal, além de invasivo, os recémnascidos necessitam do mesmo por apresentarem distúrbios respiratórios. O uso da ventilação mecânica expõe o RN ao risco de infecção a outros fatores. A não ocorrência da troca correta dos materiais periodicamente, conforme os protocolos de assistência da unidade, aumentam a possibilidade de contaminação.

Segundo Cunha et al. (2013):

As enfermeiras pontuam que existem outros fatores ligados ao atendimento neonatal que prolongam o tempo de internação e aumenta os riscos à saúde do paciente, como o uso de antibióticos. É possível identificar nas suas falas a utilização desses fármacos em amplo espectro, sem atingir o resultado esperado.

A administração de medicamentos também é um procedimento de risco, principalmente se administrados pela via endovenosa, pois se realiza o uso de técnica asséptica para evitar a contaminação da solução. Apesar da lavagem das mãos serem preconizada antes desse procedimento.

De acordo Neves et al. (2006)

Como medida de controle de infecção à higienização das mãos não é, portanto, recomendação recente. Deve ocorrer antes e após o contato com o paciente, antes de calçar as luvas e após retirá-las, entre um paciente e outro, entre um procedimento e outro, ou em ocasiões onde exista transferência de patógenos para pacientes e ambientes, entre procedimentos com o mesmo paciente e após o contato com sangue, líquido corporal, secreções, excreções e artigos ou equipamentos contaminados por esses.

\section{A infecção hospitalar neonatal no contexto do cuidado pelo (a) enfermeiro (a).}

Os desafios são inúmeros frente no controle de infecção na UTIN, os pacientes prematuros, muitos são imunodeprimidos, inaptos em diversos sistemas e órgãos e por muitas vezes nem a barreira cutânea está adequadamente presente, havendo necessidade dianto o quadro clinico de procedimentos invasivos, como tubos, cateteres e sondas, sendo porta de entrada para a aquisição de infecções, carreiando um enorme potencial de morbidade, devido ao fraco processo de desenvolvimento, uma simples infecção pode comprometer especialmente o prognóstico neuropsicomotor dessas crianças.

De acordo Pinheiro et al. (2009), retrata que :

O tempo de permanência na UTIN é um dos principais fatores de risco para colonização e infecção por germes hospitalares. $O$ contato precoce com a mãe e o aleitamento materno promove a colonização do recémnascido por microrganismos da flora materna, protegendo-o, em parte, contra a colonização pelos germes hospitalares.

Existem vários motivos para justificar o maior risco de infecção para esse grupo, o sistema imune, pouca produção de anticorpos pelo feto no útero, risco de colonização com bactérias virulentas ou resistentes a antibióticos, porque a superfície de suas mucosas ainda não tem a microflora de proteção, o aleitamento materno de prematuros é mais difícil pela imaturidade e necessidade de hospitalização mais prolongada após o nascimento, separação prejudica a formação do vínculo mãe-filho, vinculo essencial ao sucesso da amamentação

Na UTI neonatal estudada, a enfermagem limita- se à utilização do tempo para o cuidado dos pacientes e isto acaba por interferir no tempo para outras ações do cuidado ampliado, como o tempo para as conversas com a família. Lembramos que este é um momento quando as informações são fornecidas e oportunas para a promoção da integração da equipe da unidade com a família (Souza \& Ferreira, 2010)

As (os) enfermeiras (os) demonstram que a infecção neonatal traz sofrimento físico para o RN e a sua família, manifestado através do estresse provocado pelo aumento no tempo de internação, principalmente quando não há informações claras sobre o quadro do RN, e a mãe que esperava sair da maternidade com o seu filho se depara com a situação de permanência hospitalar prolongada devido ao diagnóstico infeccioso. (Cunha et al., 2013). 
Também existe uma relação direta entre tamanho da equipe e as taxas de infecção, quanto maior o quantitativo de profissionais para os pacientes, maior o risco de IRAS, assim como a superlotação do berçário.

Nesta perspectiva os desafios para o controle de infecção podem ser considerados coletivos e agrupados em: estrutura organizacional que envolve políticas governamentais, institucionais e administrativas, relações interpessoais no trabalho e normatização do serviço, batalha biológica que aborda a identificação de novos microrganismos e a ressurgência de outros, bem como a resistência aos antimicrobianos, envolvimento profissional, com enfoque para a falta de conscientização dos profissionais, adesão às medidas de controle e o comprometimento com o serviço e o paciente, capacitação profissional, destacando-se a educação continuada, epidemiologia das infecções e medidas de prevenção e controle (Cunha et al., 2013).

Na prática, os (as) enfermeiros (as) reconhecem esses desafios e as dificuldades no controle das infecções.

O maior avanço nesta área é o investimento nos recursos humanos, tais recursos requer esforços para o seu constante aprimoramento nos diferentes métodos do controle de infecção.

Ressaltamos que, embora o processo de trabalho da UTI neonatal proporcione constantes desgastes e envolva fatores que constituem obstáculos para a oferta da assistência humanizada, percebemos que trabalhadores criam estratégias para atender às necessidades dos usuários e também desenvolvem ações que facilitem suas práticas. São profissionais que, conforme expressa o dito popular, tiram leite de pedra em uma UTI neonatal (Souza \& Ferreira, 2010).

No Brasil, a evolução da Enfermagem Neonatológica é um fenômeno recente. Na maioria dos Cursos de Graduação em Enfermagem, tradicionalmente o ensino do cuidado ao recém-nascido é realizado como unidade da Disciplina de Enfermagem Obstétrica ou da Enfermagem Pediátrica. Raros são os cursos em que a Enfermagem Neonatológica é colocada no status de Disciplina e que conta com corpo docente específico. Convém lembrar que o Curso de Graduação não objetiva o ensino do cuidado ao recém-nascido de risco, devendo o mesmo ser objeto do curso de Especialização (Costa \& Padilha, 2011).

A mudança de comportamento da equipe multidisciplinar, fator somatório no controle de infecção, sendo necessária estimular os profissionais no uso de protocolos, promovendo treinamentos, debates e informações essencias., melhorando seus conhecimentos e habilidades técnicas para a prática do exercício profissional com modelos de cuidados mais seguros.

\section{Considerações finais}

Neste contexto o (a) enfermeiro (a) destaca-se por ser o (a) profissional qualificado (a) em assumir a o papel de orientador (a) e educador (a) da equipe de enfermagem e dos usuários, realizando de forma qualificada à vigilância das infecções hospitalares em neonatos, quando da realização de procedimentos invasivos, pensando em uma assistência mais segura para tal se faz necessário possuir um atendimento clinico, observador, criterioso, humanizado, reafirmando e fortalecendo a relação enfermermagem com a equipe de enfermagem, família e neonatos.

A enfermagem neonatal busca profissionais que deve está apto a resolver as situações enfrentadas na UTIN facilitando a resolução de problemas que podem acometer aos pacientes internados na unidade

A expectativa é que as informações contidas neste artigo sejam motivo de discussão e reflexão da assistência praticada na UTIN. Desse modo, poderão contribuir para a possível reflexão e embasamento da assistência prestada durante período de internamento.

A infecção hospitalar, um tema bastante polêmico e pouco difundido, sugere-se a necessidade de novos estudos envolvendo profissionais de enfermagem em pesquisas de campo.

É sabido que os (as) enfermeiros (as) desempenham três papéis básicos, quer em ambiente hospitalar. O papel assistencial é aquele que envolve ações que atendem diretamente às necessidades de assistência à saúde e de enfermagem dos pacientes e suas famílias; o gerencial que é capaz de envolver ações que decidem, correlacionam, influenciam e facilitam aquelas que afetam as atividades de outros e que visam à determinação e realização de um objetivo; pode ser uma liderança formal de enfermagem ou um papel informal assumido periodicamente por algum (a) enfermeiro (a); e o (a) de educador (a), que envolve ações adotadas para implementar estudos de modo a determinar os efeitos reais da assistência de enfermagem para aumentar a base científica da mesma.

Além disso, os artigos explicitados no presente trabalho puderam demonstrar quais as principais consequências para os pacientes e para os (as) enfermeiros (as) e profissionais da enfermagem quanto ao processo de infecção na UTIN.

O aumento dos custos com saúde, as complicações na saúde dos pacientes já debilitados e a falta de confiança nos (as) enfermeiros (as) foram apontadas como consequências nesse trabalho e devem ser sanadas 
para que a credibilidade nesta profissão esteja em alta e para que os pacientes não venham a correr sérios riscos de morte e continuem seus tratamentos da maneira mais eficaz possível.

A maior parte dos profissionais da enfermagem não se manifesta notificando o evento com medo dos processos e procedimentos cabíveis que podem fazer com que o profissional perca o emprego, porém, é de suma importância que a equipe de enfermagem juntamente com os dirigentes das instituições de saúde promova uma estratégia educativa incentivando o (a) profissional ao preenchimento do documento de notificação garantindo a este as providencias cabíveis não para punição, mas sim voltada à educação e ações preventivas.

Um dos pilares para a prevenção de eventos infecciosos é a formação adequada do profissional de enfermagem, com embasamento técnico e científico em consonância com o compromisso profissional, a sistematização da assistência de Enfermagem e o Código de ética dos Profissionais de Enfermagem.

Contudo, percebe-se a necessidade do aprimoramento das técnicas utilizadas nesse processo, além da educação continuada dos profissionais envolvidos neste processo, reestruturação do ambiente de trabalho, e sobretudo a extrema atenção quanto a organização do processo de trabalho, dando ênfase aos propósitos corretos da assistência o que gerará maior segurança aos profissionais e pacientes.

Sabe-se que para uma melhor qualidade na assistência de enfermagem, o (a) enfermeiro(a) ao detectar as falhas na assistência deve exercer seu papel de educador (a) proporcionando à equipe um desenvolvimento técnico, para que assim possa estar prevenindo possíveis danos que envolvem terapia intensiva que possam vir a interferir drasticamente no quadro clínico dos pacientes RNs.

A equipe de enfermagem tem uma responsabilidade ímpar neste contexto, porque é quem atua direta e indiretamente em todos os processos de prevenção. A responsabilidade ética e os avanços tecnológicos impõem aos profissionais de enfermagem uma busca constante de conhecimentos que sejam específicos e que estejam atualizados.

\section{Referências}

Almeida Jorge, J., \& Pereira, H. C. V. 2013. A atuação da equipe de enfermagem da unidade de terapia intensiva neonatal na prevenção e controle da infecção hospitalar. Revista Interfaces: Saúde, Humanas e Tecnologia, 1(2).

Costa, R., \& Padilha, M. I. 2011. A Unidade de Terapia Intensiva Neonatal possibilitando novas práticas no cuidado ao recém-nascido. Revista Gaúcha de Enfermagem, 32(2), 248-255.

Cunha, K. J. B., Moura, M. E. B., Nery, I. S., \& Rocha, S. S. 2013. Representações sociais de infecção neonatal elaboradas por enfermeiras [Social representations of the neonatal infection produced by nurses]. Revista Enfermagem UERJ, 21(4), 527-532.

Dal-Bó, K., Silva, R. M. D., \& Sakae, T. M. (2012). Infecção hospitalar em uma unidade de terapia intensiva neonatal do Sul do Brasil. Revista Brasileira de Terapia Intensiva, 24(4), 381-385.

Lorenzini, E., Costa, T. C. D., \& Silva, E. F. D. 2013. Prevenção e controle de infecção em unidade de terapia intensiva neonatal. Revista Gaúcha de Enfermagem, 34(4), 107-113.

Neves, Z. C. P., Tipple, A. F. V., Souza, A. C. S. , Pereira, M. S., Melo, D. S., \& Ferreira, L. R. 2006. Higienização das mãos: o impacto de estratégias de incentivo à adesão entre profissionais de saúde de uma unidade de terapia intensiva neonatal. Revista Latino-Americana de Enfermagem, 14(4), 546-552.

Pinheiro, M. S. B., Nicoletti, C., Boszczowsk, I., Puccini, D. M. T., \& Ramos, S. R. T. S.. 2009. Infecção hospitalar em Unidade de Terapia Intensiva Neonatal: há influência do local de nascimento?. Revista Paulista de Pediatria, 27(1), 6-14

Sousa Tomaz, V., Neto, F. H. C., Almeida, P. C., Maia, R. C. F., Monteiro, W. M. S., \& Chaves, E. M. C. 2011. Medidas de prevenção e controle de infecções neonatais: opinião da equipe de enfermagem. Revista da Rede de Enfermagem do Nordeste, 12(2), 271-278.

Sousa, C. M. M., da Costa Feitosa, M. D. S., Moura, M. E. B., \& Silva, A. O. 2008. Os direitos dos usuários da saúde em casos de infecção hospitalar. Revista Brasileira de Enfermagem, 61(4), 411-417.

Souza, K. M. O. D., \& Ferreira, S. D. 2010. Assistência humanizada em UTI neonatal: os sentidos e as limitações identificadas pelos profissionais de saúde. Ciência \& Saúde Coletiva, 15, 471-480. 


\section{Minicurrículo}

Bruna Rodrigues Martins de Jesus. Graduada em Enfermagem pela Faculdade Maurício de Nassau, Salvador-BA, Especialista em Unidade de Terapia Intensiva, Especialista em Instrumentação Cirpurgica, Centro cirúrgico e Central de material e esterilização, Especialista em Gestao e Auditoria em Serviços de Saúde pela Faculdade Venda Nova do Imigrante, ES, Especialista em Enfermagem do Trabalho e Saúde Ocupacional pela Universidade Cândido Mendes, São Paulo-SP.

Como citar: Jesus, B.R.M. 2020. Atuação do (a) enfermeiro (a) na prevenção e controle das infecções hospitalares na Unidade de Terapia Intensiva Neonatal. Pubsaúde, 4, a099. DOI: https://dx.doi.org/10.31533/pubsaude4.a099

Recebido: 11 nov. 2020.

Revisado e aceito: 16 nov. 2020.

Conflito de interesse: os autores declaram, em relação aos produtos e companhias descritos nesse artigo, não ter interesses associativos, comerciais, de propriedade ou financeiros que representem conflito de interesse.

Licenciamento: Este artigo é publicado na modalidade Acesso Aberto sob a licença Creative Commons Atribuição 4.0 (CC-BY 4.0). 